\title{
Night-time predation by Steller sea lions
}

\section{New insight into the feeding habits of these mammals will help conservation attempts.}

$\mathrm{M}$ easures have been taken to curtail commercial fishing of walleye pollock (Theragra chalcogramma) in Alaska in an attempt to stop the decline of its endangered population of Steller sea lions (Eumetopias jubatus). But our nighttime observations of these mammals in Prince William Sound using infrared scanning technology, combined with acoustic surveillance of their prey's behaviour, reveal that the sea lions feed exclusively on Pacific herring (Clupea pallasi), which are less abundant than pollock but are found closer to the surface at night.

Food limitation is the principal factor in the decline of Steller sea lion populations ${ }^{1-4}$. This decline could be explained by competition with commercial fisheries, as it has coincided with the growth of the pollockfishing industry, which has become one of the largest fisheries in the world, or it could be related to a change in predator-prey relationships, possibly driven by ocean climate shifts ${ }^{5}$. Central to the uncertainty surrounding the drop in the numbers of Steller sea lions is a lack of observational data on their foraging ecology. There is no quantitative information available that directly relates the foraging behaviour of these animals to the abundance of prey species.

During the winter period, nutritional stress is high. Sonar surveys ${ }^{6,7}$ of the abundance and distribution of adult Pacific herring and walleye pollock in winter have been made in Prince William Sound in Alaska since the early $1990 \mathrm{~s}^{8}$. Steller sea lions were seen during the day near herring schools, but as no foraging activity was detectable, the significance of this cooccurrence was questionable.

We complemented our sonar surveys during March 2000 with infrared scanning of the Steller sea lions. This technology, which is widely used in night-time military operations and surveillance, enabled us to monitor the animals' activity during the hours of darkness. Our system had a $27^{\circ} \times 18^{\circ}$ field of view and a spectral response of $7-14 \mu \mathrm{m}$.

The estimated herring biomass in Prince William Sound in the sonar survey of March 2000 was 7,281 metric tonnes (95\% confidence interval, 5,898-8,664). The estimate of pollock biomass was 28,277 metric tons (95\% confidence interval, 26,034-30,420). Despite the much greater abundance of pollock, the infrared system revealed that foraging by Steller sea lions was exclusively on herring and was conducted only at night. Foraging activity was intense on dense herring schools (Fig. 1).
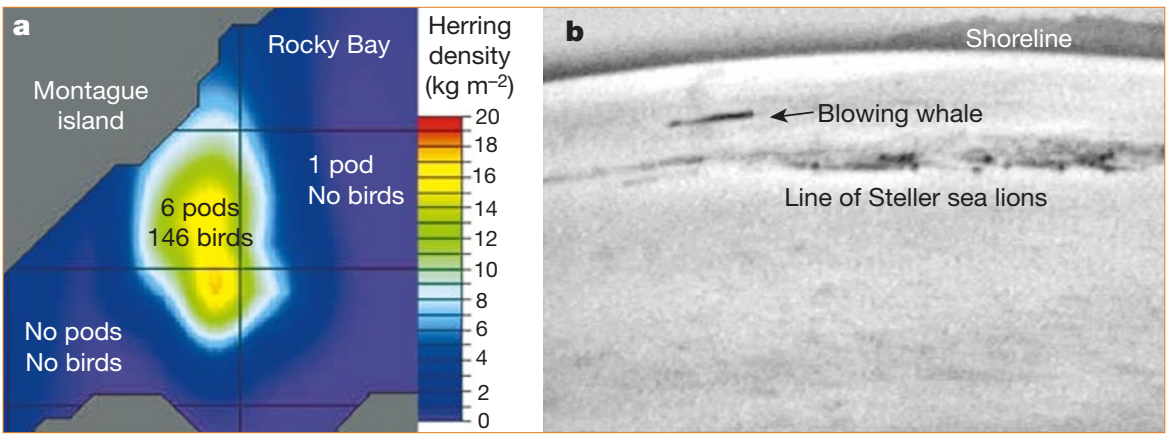

Figure 1 Location of groups (pods) of Steller sea lions around herring schools. a, Combined acoustic and infrared sensors reveal sea lions and birds located on the surface above the herring school at night in Rocky Bay, Prince William Sound (March 2000). b, Infrared video image showing a line of Steller sea lions and a humpback whale on the sea surface above a school of herring.

Steller sea lions were often observed swimming side by side in a row of 50 or more individuals along the edges of a school, suggesting that they were herding the herring. Humpback whales and seabirds were also seen to be feeding alongside the sea lions (Fig. 1). By contrast, no sea lions were coincident with pollock schools.

The sonar records revealed herring schools at depths of 10-35 m at night, but deeper during the day. Walleye pollock, on the other hand, remained at depths of over $100 \mathrm{~m}$ during both day and night. Pollock schools were also found in less protected regions and were further offshore. Although Steller sea lions are capable of dives exceeding $250 \mathrm{~m}$ (ref. 9), the more accessible distribution of herring at night may be the primary factor in the foraging behaviour of the sea lions. This distribution of herring is characteristic during an extended overwintering period in the North Gulf of Alaska.

Our results indicate that the dependence of Steller sea lions on herring as prey has been underestimated. The infrared scanning technology that has led us to this con-

\section{Genomics}

\section{Genes lost during evolution}

0 ne of the main conclusions presented by the International Human Genome Sequencing Consortium is that "hundreds of genes appear to have resulted from horizontal gene transfer from bacteria at some point in the vertebrate lineage"1. We noticed that a significant proportion of these human genes have closely related orthologues in the primitive eukaryote Dictyostelium. This observation supports independent gene loss in multiple lineages (worm, fly, yeast, plants) rather than hori- clusion should also help in the evaluation of night-time foraging behaviour of other marine mammals and seabirds, with its remarkable ability to detect individual fish flipping on the sea surface at a distance of 5-30 $\mathrm{m}$, as well as sea lions, whales and birds at over $100 \mathrm{~m}$.

Gary L. Thomas, Richard E. Thorne

Prince William Sound Science Center, PO Box 705,

Cordova, Alaska 99574, USA

e-mail: thorne@pwssc.gen.ak.us

1. National Research Council The Bering Sea Ecosystem (National Academic, Washington DC, 1996)

2. Castellini, M. Alaska Sea Grant College Program AK-SG-93-01, 4-13 (1993).

3. Merrick, R. L. et al. Can. J. Fish Aquat. Sci. 34, 1342-1348 (1997).

4. Loughlin, T. R. Biosphere Conserv. 1, 91-98 (1998).

5. Mantua, N. J. et al. Bull. Am. Meterol. Soc. 78, 1069-1079 (1997).

6. MacLennan, D. N. \& Simmonds, E. J. Fisheries Acoustics (Chapman \& Hall, London, 1992).

7. Thorne, R. E. et al. FAO Fish. Rep. 300, 217-222 (1983).

8. Thomas, G. L. et al. in Developing and Sustaining World Fisheries Resources - the State of Science and Management (eds Hancock, D. A.et al.) 606-613 (CSIRO, Collingwood, Australia, 1997)

9. Merrick, R. L. et al. Polar Res. 13, 105-114 (1994).

zontal gene transfer from bacteria.

The human genome sequence revealed 113 genes that share a high degree of identity with bacterial genes, but are absent in the completely sequenced genomes of Caenorhabditis elegans, Drosophila melanogaster, Saccharomyces cerevisiae and Arabidopsis thaliana ${ }^{1}$. Do these genes represent examples of horizontal gene transfer from bacteria to the vertebrate lineage, or were they present in both prokaryotes and early eukaryotes, but subsequently lost from all non-vertebrate eukaryotic lineages? Although this latter possibility may seem unlikely, we recently identified a gene in Dictyostelium that is clearly an orthologue of the gene that encodes soluble 
adenylyl cyclase in bacteria and vertebrates, but has not been identified in other eukaryotes $^{2}$. Dictyostelium is located in the evolutionary tree between plants and the fungi/animal crown ${ }^{3}$, and sequencing of its genome is approaching completion ${ }^{4}$ (see also http://dictybase.org).

We used all 113 listed human genes to screen for homologous sequences in Dictyostelium (27 February 2001; see supplementary information). A TBLASTN screen of the Dictyostelium database yielded 36 sequences with expectation values of less than $10^{-10}$. BLASTX analysis with the obtained Dictyostelium DNA sequences against GenBank identified 11 genes that represent clear Dictyostelium orthologues of human genes: the human sequences share a higher degree of identity with Dictyostelium than with bacterial sequences, and the bacterial sequences score more highly with respect to Dictyostelium than they do to humans (on the basis of BLAST expectation values). A further 17 Dictyostelium sequences share a high degree of identity with the human sequence, but are not obvious intermediates between the bacterial and vertebrate orthologues (see supplementary information). Thus, in at least 11 cases, the Dictyostelium and human genes have a common ancestor, eliminating the need to invoke horizontal gene transfer from bacteria.

One of the human proteins with an orthologue in Dictyostelium is monoamine oxidase (MAO). Phylogenetic analysis of this enzyme reveals a gene duplication late in the vertebrate lineage (MAO-A and $\mathrm{MAO}-\mathrm{B}$ in Fig. 1). These paralogues seem to share a predecessor with Dictyostelium, indicating that monoamine oxidase was present in early eukaryotes, and implying that the gene has been lost in worm, fly,

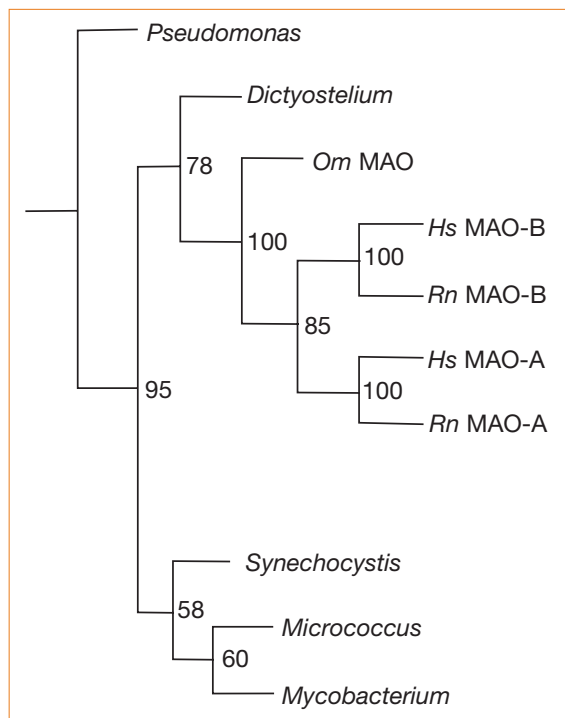

Figure 1 Phylogenetic analysis of monoamine oxidase (MAO) Numbers indicate values of bootstrap analysis $(n=100)$. Hs, Homo sapiens; Rn, Rattus norvegicus (rat); Om, Oncorhynchus mykiss (rainbow trout). plants and yeast.

Within the group of 113 genes proposed to have entered the human genome by horizontal gene transfer from bacteria, we have identified at least 11 that probably arose through normal evolution with gene loss in several lineages, suggesting that gene loss is not a rare event. With several ongoing genomic sequencing projects for lower eukaryotes, it will be interesting to see how many genes have truly undergone horizontal transfer.

Jeroen Roelofs, Peter J. M. Van Haastert Department of Biochemistry, University of Groningen, Nijenborgh 4, 9747 AG Groningen, The Netherlands

e-mail:p.j.m.van.haastert@chem.rug.nl

1. International Human Genome Sequencing Consortium. Nature 409, 860-921 (2001)

2. Roelofs, J. et al. Biochem. J. 354, 697-706 (2001).

3. Baldauf, S. L. et al. Science 290, 972-977 (2000).

4. Kay, R. R. \& Williams, J. G. Trends Genet. 15, 294-297 (1999).

Supplementary information is available at http://www.nature.com or as paper copy from the London editorial office of Nature.

\section{Bone-marrow transplantation}

\section{Failure to correct murine muscular dystrophy}

B one-marrow cells have the potential to differentiate into other cell types such as muscle fibres, and can be transplanted into acutely ${ }^{1}$ or chronically ${ }^{2}$ damaged muscle as a way of delivering normal dystrophin (the protein that is defective or missing in Duchenne's muscular dystrophy) to the skeletal and heart muscle of $m d x$ mice $^{2,3}$, an animal model for this disease. But the corrective potential of this approach has been hard to estimate against the high background of muscle fibres that spontaneously revert to synthesizing dystrophin, a feature of the original $m d x$ mutation ${ }^{4}$. Here we test the long-term efficacy of bonemarrow transplantation in a different $m d x$ mutant which is free of this problem and find that it has no impact on murine muscular dystrophy.

The $m d x 4 c v$ mutant (in which a C-to-T nucleotide transition generates a stop codon in exon 53 of the dystrophin gene) has almost no background of revertant fibres in skeletal muscle ${ }^{4}$. We sublethally irradiated (900 cGy) a group of 15 8-weekold $m d x 4 c v$ mice (C57Bl/6/Ly-5.2 background) and transplanted them with a total of $1.5 \times 10^{7}$ bone-marrow cells from a pool of 6-week-old, co-isogenic (C57Bl/6/Ly5.1) animals. We killed the mice at regular intervals from 9 weeks to 10 months after transplantation, and monitored the engraftment of donor cells by cytofluorimetric analysis of the proportion of Ly-5.1 marker compared with Ly-5.2. The degree of chimaerism averaged $85 \pm 2.7 \%$ in bone marrow (mean \pm s.e.m.), $93 \pm 1.1 \%$ in

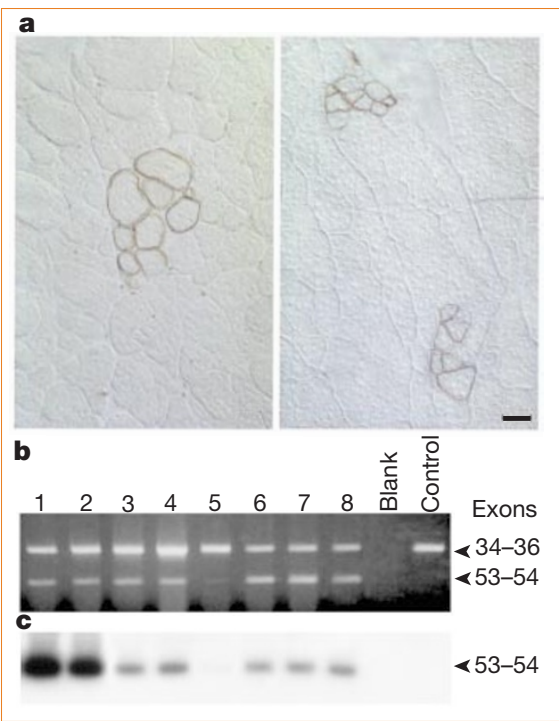

Figure 1 Expression of dystrophin in $m d x$ mice 6 months after transplantation with bone-marrow cells from co-isogenic, normal donors. a, Immunohistochemical staining of frozen sections of tibialis muscle with an anti-dystrophin monoclonal antibody. Scale bar, $50 \mu \mathrm{m}$. b, Detection of wild-type dystrophin mRNA by RT-PCR amplification using specific primers for exons 53 and 54 in samples of total RNA extracted from skeletal muscle of transplanted $m d x$ mice (lower bands). A fragment encompassing exons 33-36 in both wild-type and mutant dystrophin RNA is amplified as an internal control (upper bands). Lanes 1-8, samples from transplanted mice; 'blank', PCR assay without RNA; 'control', mock-transplanted $m d x$ control. c, Southern-blot hybridization with an internal, specific oligonucleotide probe for exon 53.

spleen, $92 \pm 2.9 \%$ in thymus and $94 \pm 0.8 \%$ in peripheral blood throughout the followup study.

We counted dystrophin-positive $\left(\right.$ dys $\left.^{+}\right)$ fibres in histological sections of representative muscles (tibialis anterior, quadriceps, diaphragm) after immunohistochemical staining with an anti-dystrophin antibody in transplanted and age-matched, mocktransplanted, control $m d x 4 c v$ mice. Clusters of dys $^{+}$fibres were apparent in muscle sections of transplanted animals, averaging $0.23 \pm 0.05 \%$ (minimum, $0.06 \%$; maximum, $0.54 \%$ ) throughout the 10 -month study (Fig. 1a). The proportion of $\mathrm{dys}^{+}$ fibres in control animals averaged $0.14 \pm 0.03 \%$ (minimum, $0.02 \%$; maximum, $0.33 \%)$, a statistically significant difference $(F=5.99, P=0.02)$. In neither group was there any significant increase in the number of dys ${ }^{+}$fibres in young (under 5 months) and old (over 12 months) animals. The average number of fibres contained in each dys ${ }^{+}$cluster varied from 3 to 30 , with no significant change with age in either group.

To demonstrate the presence of normal dystrophin in the muscle of transplanted mice (the antibody does not distinguish between corrected and revertant fibres), we developed a polymerase chain reaction with reverse transcription (RT-PCR) assay to distinguish wild-type dystrophin messen- 
differential gametic imprinting, as well as on the amount of gene product needed for biological function.

Dolf Weijers*, Niko Geldner $\dagger$, Remko Offringa ${ }^{\star}$, Gerd Jürgens $\dagger$ ${ }^{*}$ Institute of Molecular Plant Sciences, Leiden University, 2333 AL, Leiden, The Netherlands. $\dagger$ Zentrum für Molekularbiologie der Pflanzen, Universität Tübingen, 72076 Tübingen, Germany

e-mail: gerd.juergens@zmbp.uni-tuebingen.de

1. Surani, M. A. et al. Development (suppl.) 89-98 (1990).

2. Lehmann, R. \& Nüsslein-Volhard, C. Dev. Biol. 119, 402-417 (1987)

3. Vielle-Calzada, J.-P., Baskar, R. \& Grossniklaus, U. Nature 404, 91-94 (2000).

4. Lu, P., Porat, R., Nadeau, J. A. \& O’Neill, S. D. Plant Cell 8, 2155-2168 (1996).

5. Springer, P. S., Holding, D. R., Groover, A., Yordan, C. 8 Martienssen, R. A. Development 127, 1815-1822 (2000).

6. Mayer, U., Büttner, G. \& Jürgens, G. Development 117, 149-162 (1993).

7. Waizenegger, I. et al. Curr. Biol. 10, 1371-1374 (2000).

8. Hamann, T., Mayer, U. \& Jürgens, G. Development 126, 1387-1395 (1999).

9. http://www.plantsci.cam.ac.uk/Haseloff/GAL4/mGAL4.html

10. Thoma, S. et al. Plant Physiol. 105, 35-45 (1994).

Vielle-Calzada et al. reply - Our results, based on a study of 20 loci, indicate that the contributions by the maternal and paternal genome to early seed development in Arabidopsis are not equivalent, as evidenced by a lack of detectable paternal gene activity during the first few divisions after fertilization. As these loci are distributed throughout the genome, we inferred that early embryo and endosperm development are mainly under maternal control, but this may not be true for every locus and, as in X-chromosome inactivation ${ }^{1}$, we would expect some loci to escape this silencing mechanism. We did not claim that maternal control is complete, but suggested that the activity of many genes during early embryo and endosperm formation could depend solely on transcription of the maternally inherited allele before and/or after fertilization.

Previously, early seed formation was thought to involve transcription from both parental copies immediately following fertilization, and maternal effects were considered rare or non-existent ${ }^{2}$. The time at which paternal activity can first be detected, however, is likely to vary from embryo to embryo and from gene to gene in different nuclei, as in Drosophila ${ }^{3}$. Weijers et al. report paternal expression of AtRPS5A::GUS as early as the two-cell stage, confirming that transcription in the zygote is not the rule for paternally inherited alleles, whereas transcription from maternal alleles has been demonstrated immediately after fertili-zation of the central cell ${ }^{4}$. We do not know what percentage of embryos show early AtRPS5A::GUS expression, nor the relative paternal and maternal activity, but there may also be less pronounced parentof-origin differences.
New evidence supporting the non-equivalence of maternal and paternal genomes during early seed development is based on experiments with reporter genes ${ }^{5-8}$ and genetic assays revealing maternal effects of genes thought to act purely zygotically ${ }^{6}$ (S. Gilmore and C. Somerville, personal communication; J. Moore and U. G., unpublished results). Whether and at what stage expression of the paternal allele is sufficient for normal development will depend on the level of activity required for gene function. In a two-component transactivation system, no paternal activity was found during early seed development using $p O p:: G U S$ reporter lines with several activator lines. Some early defects were evident with a $p O p:: B A R N A S E$ reporter, however, suggesting that paternal transcription is very low but is sufficient to cause $B A R$ NASE-induced defects in some embryos ${ }^{8}$. These results confirm the non-equivalence of maternal and paternal contributions to early seed development. Like imprinted genes in mammals, this difference is probably not absolute and may be due to different levels of maternal and paternal transcripts.

Our titration experiments indicated a difference in transcript levels of at least 80 -fold for genes we tested by PCR. Weijers et al. report an expression difference in reciprocal crosses with UAS:GUS at the heart to torpedo stage (Fig. 1d), when we showed that both parental alleles are active at other loci we tested; indeed, this differential expression translates into an absence of detectable paternal activity at earlier stages using the $p O p:: G U S$ reporter system ${ }^{8}$. For some genes, such as KEULE or KNOLLE, low paternal expression may be sufficient for normal development, although very early defects (such as developmental delay) that are rescued by a paternal wild-type allele may be difficult to detect by scoring multinucleate

\section{corrections}

\section{Night-time predation by Steller sea lions}

G. L. Thomas, R. E. Thorne

Nature 411, 1013 (2001)

We stated that our acoustic surveys in Prince William Sound since 1993 and infrared surveys since 2000 suggested that these sea lions "feed exclusively" on herring. However, it has been drawn to our attention that this statement is misleading. In clarification, the sea lions were selectively targeting the relatively shallow (0-50-m depth) schools of Pacific herring (Clupea pallasi) at night as a source of winter forage to the exclusion of relatively larger and deeper (150-250 m) concentrations of walleye pollock.

\section{Transatlantic robot-assisted telesurgery}

J. Marescaux, J. Leroy, M. Gagner, F. Rubino, D. Mutter,

M. Vix, S. E. Butner, M. K. Smith

Nature 413, 379-380 (2001) embryos. Moreover, rescue of an early embryonic phenotype by a paternal wildtype allele provides no evidence against differences in parental transcript levels.

Although the exact time of paternal activation was not central to our report, most evidence so far suggests that no consistent paternal gene activity can be detected in the embryo or endosperm for several cell divisions. The results of Weijers et al. do not contradict our findings, but instead represent possible exceptions to a general rule. Specific genes that are important during early development (for example, those involved in cytokinesis that are distinctly regulated in the female gametophyte and the zygote ${ }^{9}$ ) may be under selection for earlier expression and be specifically activated early in development. Further investigation is required into how common early-expressing paternal genes are, and how maternal and paternal expression differs quantitatively.

Jean-Philippe Vielle-Calzada ${ }^{\star}$, Ramamurthy Baskar $\dagger$, Ueli Grossniklaus $\dagger$

${ }^{*}$ Cinvestav-Plant Biotechnology Unit, Irapuato,

Mexico; $†$ Institute of Plant Biology, University of

Zürich, 8008 Zürich, Switzerland

e-mail:grossnik@botinst.unizh.ch

1. Goto, T. \& Monk, M. Microbiol. Mol. Biol. Rev. 62, 362-368 (1998).

2. Howell, S. H. Molecular Genetics of Plant Development (Cambridge Univ. Press, Cambridge, 1999).

3. Pritchard, D. K. \& Schubiger, G. Genes Dev. 10, 1131-1142 (1996).

4. Vielle-Calzada, J.-P. et al. Genes Dev. 13, 2971-2982 (1999).

5. Luo, M., Bilodeau, P., Dennis, E. S., Peacock, W. J. \&

Chaudhury, A. M. Proc. Natl Acad. Sci. USA 97, 10637-10642 (2000).

6. Springer, P. S., Holding, D. R., Groover, A., Yordan, C. \& Martienssen, R. A. Development 127, 1815-1822 (2000).

7. Sorensen, M. B., Chaudhury, A. M., Robert, H., Bacharel, E. \& Berger, F. Curr. Biol. 11, 277-281 (2001).

8. Baroux, B., Blanvillain, R. \& Gallois, P. FEBS Lett. 509, 11-16 (2001).

9. Mayer, U., Herzog, U., Berger, F., Inzé, D. \& Jürgens, G. Eur. J. Cell Biol. 78, 100-108 (1999)

The correct address of the third author of this communication is Division of Laparoscopic Surgery at Mount Sinai School of Medicine and Mount Sinai Medical Centre, New York 10029, USA.

Peptide antibiotics in mast cells of fish

Umaporn Silphaduang, Edward J. Noga

Nature 414, 268-269 (2001)

The concentrations listed in Table 1 are in $\mu \mathrm{g} \mathrm{ml}^{-1}$.

\section{erratum}

\section{Nitrate flux in the Mississippi River}

G. F. Mclsaac, M. B. David, G. Z. Gertner, D. A. Goolsby Nature 414, 166-167 (2001).

In Fig. 1 of this communication, the line referred to as "black" is in fact blue; also, in the fourth line of the third column, $P$ should be greater than 0.05 . 